\title{
LA MÁQUINA DEL TIEMPO
}

The time machine

A máquina de tempo

\section{Laura Álvarez Cañas}

Centro Rural Agrupado Ruta de la Plata, León, España. Teléfono: +34 676676655. Correo electrónico: guasariiii@hotmail.com

\section{Resumen}

El proyecto de trabajo titulado "la máquina del tiempo" pretende mostrar por un lado, cómo de manera lúdica y alternativa a la pedagogía tradicional, los alumnos desde el área de Ciencias Sociales conocen, comprenden y afianzan los contenidos relativos a las etapas de la historia de la humanidad, permitiéndoles la adquisición de aprendizajes significativos. Para ello, en primer lugar a través de un juego deberán ser capaces de ordenar en el tiempo aquellos acontecimientos de la historia más importantes para posteriormente realizar un pequeño trabajo a modo de "lapbook" con ellos, fomentándose los procesos de evaluación formativa. Por otro lado, deberán elaborar su propia historial personal, resaltando aquellos hechos más importante ocurridos a la lo largo de su vida. Cabe destacar que tanto en la historia social como personal, no solamente se tendrán en cuenta los aspectos relativos al pasado, sino también al presente $\mathrm{y}$ al futuro.

Palabras clave: historia personal y social; evaluación formativa; aprendizaje significativo

\begin{abstract}
The experience of the time machine aims to show, on the one hand, how in a playful and alternative way to traditional pedagogy, students from the area of Social Sciences know, understand and consolidate the contents relating to the stages of history of humanity, allowing them to acquire meaningful learning. To do this, first through a game should be able to order in time those events of the most important history to later perform a small work as a "lapbook" with them, encouraging the processes of formative evaluation. On the other hand, they must develop their own personal history, highlighting those most important events that occurred throughout their lives. It should


be noted that in social and personal history, not only aspects relating to the past but also to the present and to the future will be taken into account.

Keywords: personal and social history; formative assessment; meaningful learning

\section{Resumo}

O projecto de documento intitulado "Time Machine" pretende mostrar, por um lado, como forma divertida e uma alternativa à pedagogia tradicional, estudantes da área de ciências sociais conhecer, entender e reforçar os conteúdos relativos às etapas da história da humanidade, permitindo a aquisição de aprendizagens significativas. Para fazer isso, primeiro através de um jogo que deve ser capaz de ordenar no tempo desses eventos história mais importante mais tarde para fazer um pequeno trabalho como um "lapbook" com eles, estimulando processos de avaliação formativa. Por outro lado, eles devem desenvolver a sua própria história pessoal, com destaque para os eventos mais importantes para toda a sua vida. Notavelmente, tanto a história social e pessoal, não só os aspectos do passado, mas também o presente eo futuro são levados em conta.

Palavras-chave: história pessoal e social; avaliação formativa; aprendizagem significativo

\section{Introducción}

A lo largo de la Educación Primaria, desde el área de Ciencias Sociales se ven las características más relevantes de las etapas de la Historia, desde la Prehistoria hasta llegar a la Edad Contemporánea. El proyecto de trabajo "La máquina del tiempo" surge con la idea de llevar a cabo un trabajo global al finalizar la etapa, en el que no solamente deben manifestar aquellos conocimientos adquiridos al respecto, sino que también deben ser capaces de mostrar su pequeña historia personal. De esta forma, comprobarán cómo la evolución de la sociedad está marcada por el transcurso del tiempo y los acontecimientos que ocurren a su paso. Igualmente, verán qué cambios han experimentado ellos mismos desde que han nacido hasta la actualidad, siendo capaces de poder dividir su vida en determinados períodos, ya que, siguiendo a Pagés y Santisteban (2010), el aprendizaje del tiempo histórico debe basarse en las relaciones entre pasado, presente y futuro, a nivel personal y social. Además, no sólo debemos enseñar una determinada periodización, sino que también debemos enseñar a periodizar 
(Ferro, 1991). Potenciándose, de esta forma, la construcción de aprendizajes significativos (Ausubel, 1968).

\section{Contextualización}

La experiencia ha sido llevada a cabo durante el curso 2015-2016 en una localidad perteneciente al Centro Rural Agrupado "Ruta de la Plata", en la provincia de León. El aula estaba formada por ocho alumnos de diferentes niveles de las etapas educativas de Infantil y Primaria (uno de $3^{\circ}$ de Infantil, uno de $1^{\circ}$ de Primaria, tres de $4^{\circ}$ y tres de $6^{\circ}$ curso). Por razones obvias, justificadas en la introducción, el proyecto se llevó a cabo en el último curso de la etapa de Primaria desde el área de Ciencias Sociales. El ritmo y el estilo de aprendizaje de los alumnos son muy buenos, presentando una actitud positiva hacia las tareas propuestas.

\section{Diseño y desarrollo}

Para llevar a cabo el proyecto, lo primero que se realizó fue una caja decorada (Figura 1) a modo de máquina del tiempo, en su interior se dividió en tres partes: pasado, presente y futuro.

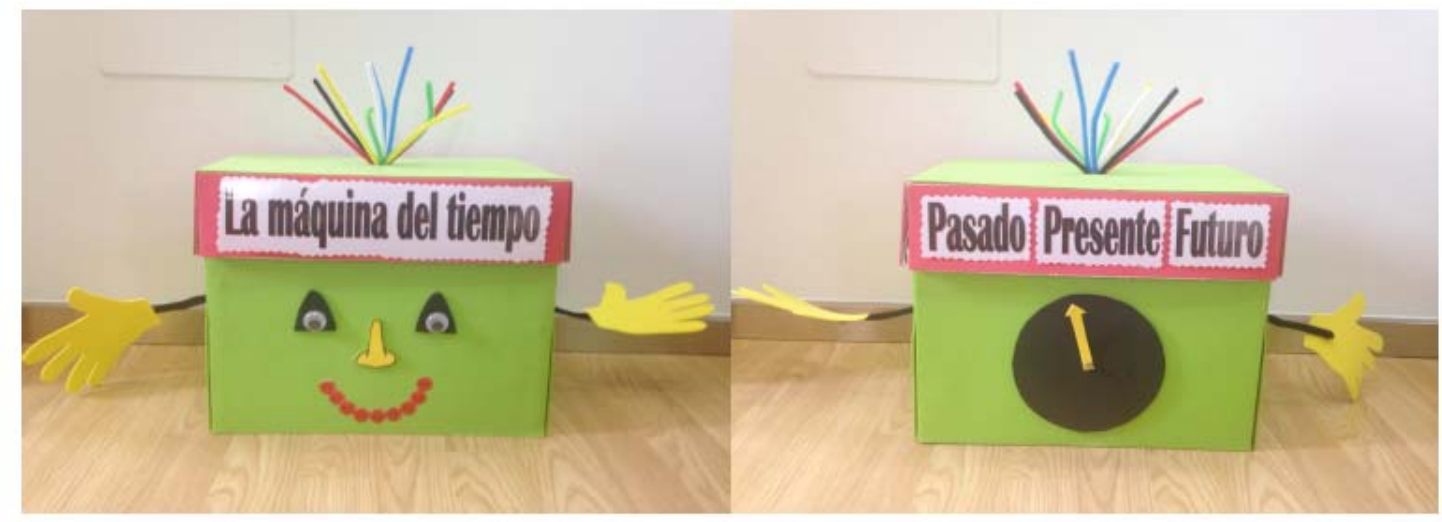

Figura 1.

Máquina del tiempo (elaboración propia, 2016)

Estructuramos la experiencia en tres momentos:

- En primer lugar, se planteó un sencillo juego, en la zona del pasado se introdujeron unas tarjetas con los acontecimientos más importantes ocurridos a lo largo de la Historia que debían ubicar de forma cronológica en la época correspondiente (Figura 2). Para ayudarles a jugar cuentan con tres comodines cuando no saben o dudan con algún hecho: a) Mirar en la carpeta del profesor donde aparecen todas las 
tarjetas ordenadas; b) Buscar en internet y c) Pedir ayuda al compañero. Pueden utilizar cada comodín un máximo de dos veces cada uno. A continuación, en la zona del presente debían realizar ellos mismos unas tarjetas con los hechos relevantes acontecidos en el año 2016; para posteriormente hacer lo propio en la zona de futuro con aquellos sucesos que iban a ocurrir en un margen de 10 años aproximadamente.

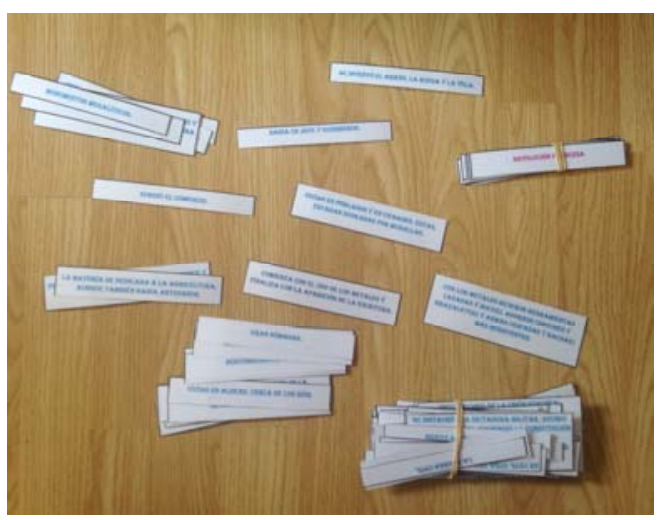

Figura 2.

Tarjetas (elaboración propia, 2016)

- En segundo lugar, debían elaborar un lapbook, es decir, un libro con solapas realizado con cartulina con la información relevante obtenida en el juego anterior (Figura 3).

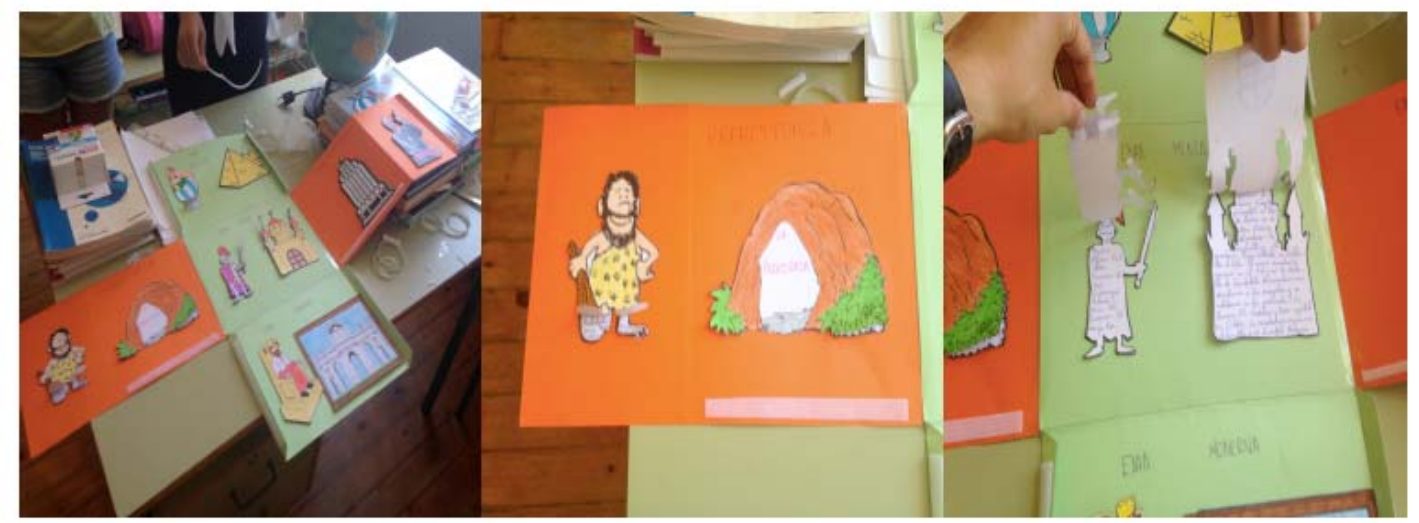

Figura 3.

Lapbook (elaboración propia, 2016)

- Por último, cada alumno debía elaborar su propia máquina del tiempo, constatando los hechos más importantes ocurridos en su "pequeño" pasado, qué le estaba ocurriendo en el presente y cómo se veía él con 25 años aproximadamente. Otra 
opción (aunque no puesta en práctica) podría ser que una vez realizada cada uno escribiera su propia biografía.

\section{Evaluación}

A la hora de llevar a cabo la evaluación de la experiencia se tuvieron en cuenta los tres momentos de la misma. Los alumnos deberán autoevaluar su propio trabajo. Así, el hecho de que participen activamente en su propia evaluación implica una mayor responsabilidad por su parte, siendo conscientes de qué aspectos han realizado bien y aquellos que deben resolver para poder mejorar su aprendizaje. Aquí cobra especial relevancia el concepto de evaluación formativa (López-Pastor et al., 2006) ya que, a través de la misma, se pretende mejorar y optimizar los procesos de enseñanzaaprendizaje.

A continuación, en el lapbook, deberán tener en cuenta dos aspectos: a) La presentación: los márgenes, la claridad, la limpieza, el estilo y la originalidad; y b) El contenido: si todos los apartados aparecen correctamente. A continuación se muestra una rúbrica de autoevaluación para los alumnos de $6^{\circ}$ modificada de Casado (2014) (Figura 4).

\begin{tabular}{|c|c|c|c|}
\hline \multicolumn{2}{|l|}{ VALORACIÓN DE LA PRESENTACIÓN } & \multicolumn{2}{|l|}{ VALORACIÓN DEL CONTENIDO } \\
\hline \multicolumn{2}{|l|}{ Márgenes } & $\begin{array}{l}\text { Muy completo: están todos los apartados } \\
\text { desarrollados adecuadamente. }\end{array}$ & 5 \\
\hline Se respetan en todo momento. & 2 & Completo: un apartado no está desarrollado & 3 \\
\hline Se respetan a menudo, aunque también hay olvidos. & 1 & adecuadamente. & \\
\hline No se respetan (es generalizado) & 0 & Incompleto: falta un apartado. & 0 \\
\hline \multicolumn{2}{|l|}{ Claridad } & \multirow[t]{4}{*}{ Observaciones: } & \\
\hline El desarrollo y títulos están separados adecuadamente. & 2 & & \\
\hline A veces, aparece todo demasiado junto. & 1 & & \\
\hline Todo el trabajo parece demasiado junto y amontonado. & 0 & & \\
\hline \multicolumn{2}{|l|}{ Limpieza } & \multicolumn{2}{|l|}{2} \\
\hline Hojas cuidadas y limpias (sin arrugar, sin borrones, etc.) & 2 & \multirow{2}{*}{\multicolumn{2}{|c|}{ VALORACIÓN GENERAL }} \\
\hline Hojas cuidadas (hay algún fallo tachado discretamente) & 1 & & \\
\hline Hojas ni cuidadas, ni limpias (borrones, tachaduras, etc.) & 0 & & \\
\hline \multicolumn{2}{|l|}{ Títulos } & & \\
\hline Aparecen reflejados siempre. & 2 & & \\
\hline Falta alguno por reflejar. & 1 & & \\
\hline No hay ninguno en el trabajo. & 0 & & \\
\hline \multicolumn{2}{|l|}{ Estilo y originalidad } & & \\
\hline La presentación es original y creativa, con personalidad. & 2 & & \\
\hline La presentación es sencilla, pero buena. & 1 & & \\
\hline La presentación es muy sosa y anonadina. & 0 & & \\
\hline Observaciones: & & & \\
\hline
\end{tabular}

Figura 4.

Rúbrica de evaluación y calificación del lapbook (modificada de Casado, 2014) 
En último lugar, en la elaboración de la propia caja, se tendrán en cuenta aspectos como el estilo y la originalidad en la presentación de la misma. Además, deberán exponer su trabajo ante la clase, valorándose aspectos relativos a la expresión oral, elemento que debe ser desarrollado transversalmente desde las áreas de Educación Primaria, como por ejemplo la existencia de un guion previo o la fluidez en la exposición (RD 126/2014. Art.10)

En cuanto a la evaluación de la experiencia por parte de los alumnos, se llevó a cabo a través de intercambios orales, donde comentaron lo divertida que había sido, sobretodo el hecho de elaborar su propia máquina del tiempo, ya que conocieron muchas anécdotas de cuando eran pequeños. También, se reflejó en el diario del profesor, la alta participación de los alumnos durante el desarrollo de la experiencia, preguntando siempre que tenían cualquier duda

\section{Conclusiones}

Lo más reseñable fue la motivación de los alumnos al realizar estos trabajos alternativos a la enseñanza tradicional. Como van siendo partícipes de su proceso de aprendizaje, de forma lúdica y distendida, afianzándose espontáneamente las relaciones sociales al llevar a cabo la actividad con sus compañeros. Además, en este caso, la participación es doblemente motivadora, ya que son ellos, los que tienen que narrar a sus compañeros su historia personal. Como propuestas de mejora, los alumnos podrían realizar su propia biografía de forma escrita, así como incluir dentro del "lapbook" una línea del tiempo con los acontecimientos destacados.

\section{Referencias}

Ausubel, D. (1968). Procesos psicológicos básicos. Madrid: Laia.

Casado, O. (2014). Rúbrica para la valoración de los cuadernos de trabajo personal Recuperado de https://drive.google.com/file/d/0B7xEdP1O4dj8Z3VrSlZOQXdNYm8/view

Ferro, M. (1991) Visions de l'histoire et périodisation, une typologie. En AA.VV., Périodes. La construction du temps historique. Actes du Ve Colloque d'histoire au present (pp. 99 - 101). Paris: Éditions de l'École des Hautes Études en Sciences Sociales et Histoire au présent. 
López-Pastor, V. M. (Coord.) (2006). La Evaluación en Educación Física: Revisión de los modelos tradicionales y planteamiento de una alternativa: La evaluación formativa y compartida. Buenos Aires: Miño y Dávila.

Pagès, J., \& Santisteban, A. (2010). La enseñanza y el aprendizaje del tiempo histórico en la Educación Primaria. Cedes 30(82), 281-309

Real Decreto 126/2014, de 28 de febrero, por el que se establece el currículo básico de la Educación Primaria (BOE $n^{\circ} 52$, de 1 de marzo). 